Short Notes

\title{
Brasile, terra del futuro
}

\author{
GIOVANNA CAMPANI²
}

Riassunto. La breve nota che segue rappresenta un carnet di viaggio, redatto tra fine ottobre 2020 e fine gennaio 2021, nello stato brasiliano del Ceara ed a Rio de Janeiro. Comprende brevi descrizioni di squarci di vita quotidiana, nella vaga ricerca di uno sguardo antropologico; riflessioni o piuttosto tentativi di elaborare un'embrione di analisi; confuse evocazioni di sentimenti e ricordi. Rispetto al tema della pandemia, che non può essere ignorato in questo periodo, la nota constata come il distanziamento sociale non possa essere praticato, ma questo non implica un maggior numero di morti rispetto all'Europa (al contrario).

\begin{abstract}
The short note represents a travel "journal", prepared between the end of October 2020 and the end of January 2021, in the Brazilian state of Ceará and in Rio de Janeiro. It includes brief descriptions of glimpses of everyday life, in the vague search for an anthropological gaze; reflections or rather attempts to develop an embryonic analysis; confused evocations of feelings and memories. The pandemic cannot be ignored, but the note shows how social distancing cannot occur in Brazilian society. Despite this, Brazil does not have a higher number of deaths than European countries, but lower.
\end{abstract}

\section{Introduzione}

Le pagine che seguono rappresentano un carnet di viaggio, redatto tra fine ottobre 2020 e fine gennaio 2021, nello stato brasiliano del Ceará e nella città di Rio de Janeiro. Comprende brevi descrizioni di squarci di vita quotidiana, nella vaga ricerca di uno sguardo antropologico; riflessioni o piuttosto tentativi di elaborare unembrione di analisi; confuse evocazioni di sentimenti e ricordi.

Nell'ottobre 2020, il mio viaggio in Brasile era in programma da più di un anno, per svolgere alcune ricerche sulla vita e le musiche di Chiquinha Gonzaga. Era in corso una pandemia, ma neppure la peste bubbonica mi avrebbe fatto desistere dal progetto. I nostri antenati non continuavano a muoversi anche in tempi di peste, praticamente endemica tra il 1500 ed il 1600? Forse che Michel De Montaigne rinunciò ad intraprendere il suo viag-

\footnotetext{
${ }^{1}$ Brasile, terra del futuro, Brasil, Land von Zukunft è una delle ultime opere dello scrittore austriaco, Stefan Zweig, che, in fuga dall'Europa, vi trascorse gli ultimi anni della sua vita, a Petropolis, vicino a Rio de Janeiro. Scritto fra il Brasile e New York nel '41, Brasile. Il paese del futuro passa in rassegna la storia del paese dal 1500 fino al 1940, la sua economia e la cultura, fornendone una visione piena di speranza per il futuro e modello per l'intera umanità, per l'accettazione ed anzi l'esaltazione del meticciato.

${ }^{2}$ Catedrática de Educación Intercultural y Antropología de Género en la Universidad de Florencia. Email: giovanna.campani@unifi.it
}

Copyright $\odot 2021$ The Author(s). Open Access. This in an open access article published by Firenze University Press (www.fupress.net/index.php/ccselap) and distributed under the terms of the Creative Commons Attribution 4.0 International License. The Creative Commons Public Domain Dedication waiver applies to the data made available in this article, unless otherwise stated. 
Figura 1. Lapide del caffè Brasile.

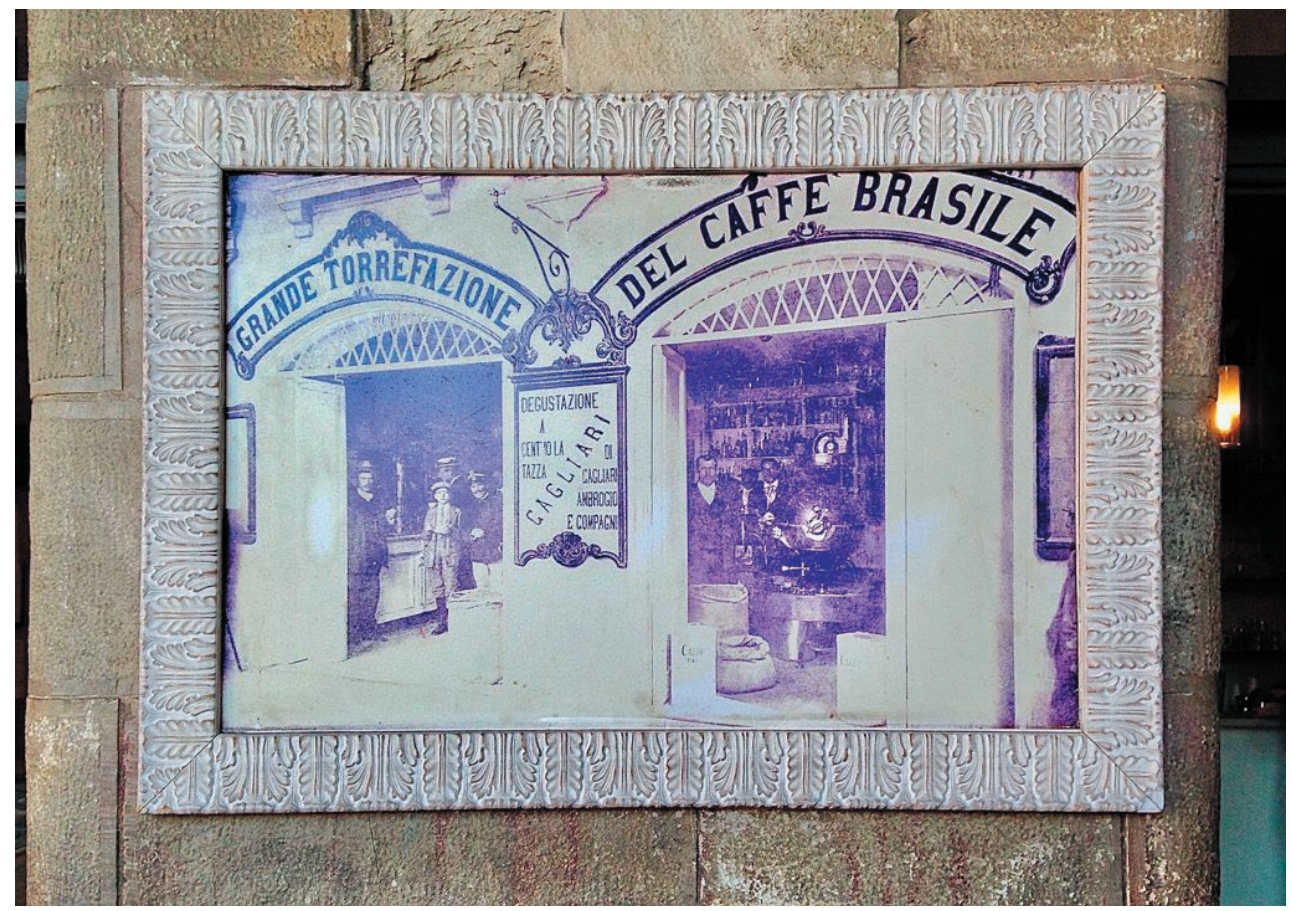

gio in Italia attraverso la Germania e la Svizzera nel 1580-81, perché c'era un focolaio di peste a Genova?

Aggiungo che io ho una relazione familiare con il Brasile: vi ritorno per inseguire il ricordo dei racconti ascoltati durante l'infanzia sulla vita di nonno Rodolfo, piantagioni, canna da zucchero, serpenti, foreste del Mato Grosso, cavallette, battaglie di galli, profumi di caffè. Il tutto trasportato in Italia via Marsiglia nel 1912, con l'apertura del Caffè IL BRASILE affacciato sulla Piazza Grande della mia città natale, oggi commemorato da una lapide.

\section{Padre Cicero}

Lascio l'Europa inviluppata nell'autunno grigio dei lockdown, popolata da fantasmi mascherati che strisciano veloci lungo i muri, per evitare incontri ravvicinati. Ritrovo la vita nel grande mercato di Fortaleza, discutendo a lungo con uno scultore di statue sacre (con mio disappunto si tratta di santi e madonne cristiani, non di "orixas"3 che lartista popolare conosce poco, a parte Yemaja). Alla fine acquisto un piccolo Padre Cicero, che é, mi assicura lo scultore "muito milagroso"4, mi porterà fortuna e farà si che i miei desideri si realizzino.

\footnotetext{
${ }^{3}$ Orixás sono divinità delle religioni afrobrasiliane, il candomblé e l'umbanda.

4 "Cícero Romão Batista ( $\underline{1844}$ - Crato-, $\underline{1934}$ Juazeiro do Norte) fu un sacerdote cattolico brasiliano. Nel-
} 
Figura 2. Statua di Padre Cicero.

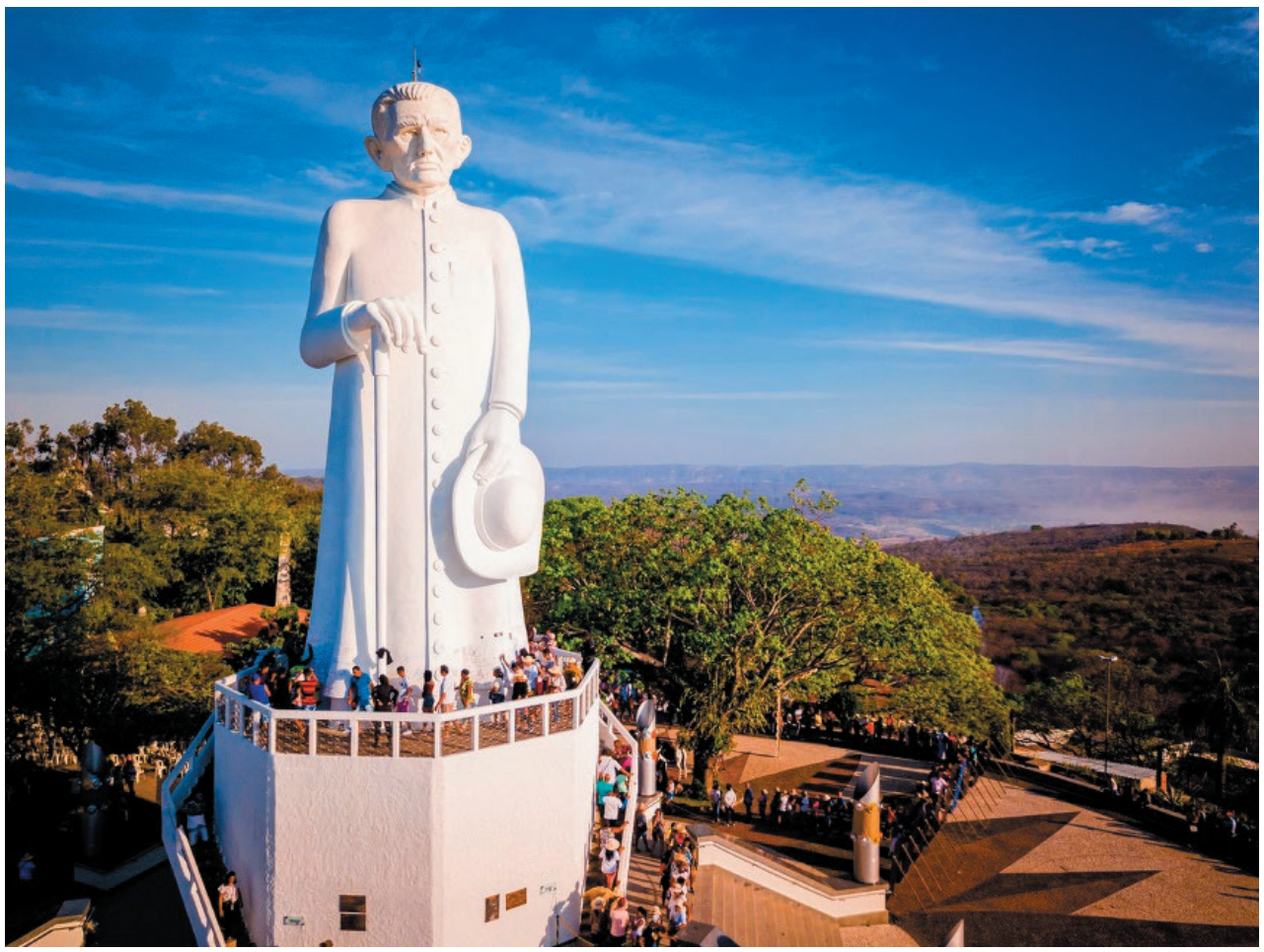

Curiosa figura quella di Padre Cicero! Nato nel 1844 a Crato, Ceará, Nordeste, ordinato sacerdote nel 1870, vicario della cittadina di Juazeiro do Norte, cacciato dagli ordini per via di un presunto miracolo in tempo di siccità (un'ostia che sanguinava), senza mai smettere la sottana da prete iniziò una carriera di politico e combattente ${ }^{5}$. Da vicario divenne prefetto di Juazeiro, dove fece costruire un aeroporto, aprì diverse scuole, tra cui la Scuola Normale Rurale, stimolò l'agricoltura, aiutò i poveri. E poiché in tempo di guerre civili, la politica non si limita al confronto verbale, Padre Cicero partecipò a rivolte contro il governo federale, con un esercito di cangaceiros $^{6}$ e di pelligrini (la storia del Nordeste brasiliano è un'affascinante epopea di rivolte guidate da banditi e da profeti ispirati che ricordano i dolciniani ${ }^{7}$ medioevali).

Due milioni e mezzo di persone visitano ogni anno la statua di Padre Cicero, alta 27 metri, eretta nella Serra do Catolé, sulla Colina do Horto nel 1969. Vorrei capirne di più, di questa religiosità popolare verso Padre Cicero: esistono connessioni con la teologia della liberazione?

la devozione popolare è conosciuto come Padre Cicero o Padim Ciço. Carismático, godé di grande prestígio e influenza sulla vita sociale, politica e religiose del Ceará e del Nordeste.

${ }^{5}$ https://www.ebiografia.com/padre_cicero/

${ }^{6}$ bandit \{subst.\} a 'social bandit' from the Northeast of Brazil, often supported by poor rural peasants \{subst.\}

${ }^{7}$ https://it.wikipedia.org/wiki/Dolciniani 


\section{O Por do Sol}

Allons voir un coucher de soleil... Così scrive Saint Exupéry.

La statua di Padre Cicero mi accompagna nel viaggio lungo la splendida costa del Céara, verso Guajiru e poi Jericoara (Jeri), villaggio di pescatori incastonato tra dune bianche e mare scintillante, in perpetuo movimento tra alta e bassa marea. Un vento perenne soffia nelle vele dei kite surfers, per i quali Jericorara è un paradiso.

A causa della pandemia, a Jeri, gli europei sono oggi minoritari. Quelli presenti sono fuggiti dalle ripetute restrizioni imposte nei loro paesi (ristoranti chiusi, bar chiusi, cinema chiusi). I tedeschi, gli svizzeri, gli austriaci discutono della Corona-Wahnsinn ${ }^{8}$, tra una capirinha e un açai. Qualcuno resterà: per quanto in via di declassamento, gli europei possono ancora procurarsi un permesso di soggiorno senza troppe difficoltà. Non sono ancora costretti a chiedere l'asilo politico.

I turisti oggi sono soprattutto brasiliani, venuti a festeggiare il Dia da consciencia negra ${ }^{9}$, il 20 di novembre, che celebra Zumbi, eroe della resistenza contro la schiavitù, vissuto nel secolo $\mathrm{XVII}^{10}$. Mi chiedo quanti tra i turisti europei conoscono la storia dei Quilombos, le comunità autogestite di schiavi fuggiaschi, a cui si univano indios, marginali, fuggiaschi. La fuga verso la libertà. Sarebbe unoccasione per riflettere sugli orrori del colonialismo, ma anche per riflettere sulla fragilità di qualsiasi condizione- di fronte al potere.

A Jeri la vita e la socialità pulsano ovunque, sulla spiaggia, nei Quiosquinos che preparano meravigliosi spiedini di aragosta, nei mercatini. Poi alla sera, Jeri diventa una comunità che svolge un rituale collettivo: il pellegrinaggio sulle dune al momento del tramonto, O Por do Sol. "J'aime bien les couchers de soleil. Allons voir un coucher de soleil..." sussurra le Petit Prince."Ah! petit prince, j’ai compris, peu à peu, ainsi, ta petite vie mélancolique. Tu n'avais eu longtemps pour distraction que la douceur des couchers de soleil."

Ho scoperto, durante questo viaggio, che i brasiliani hanno la tenera abitudine di contemplare i tramonti e si ritrovano nei luoghi da cui la vista è migliore.

Sulle orme del Petit Prince, a Jeri, ho scoperto che si può assistere alla Por do Sol de Jeri per quarantatré volte senza mai stancarsi dello spettacolo, incantandosi davanti alla sfumatura di una nuvola. Lo spettacolo del cielo al tramonto sopra Jeri ha compensato tutti i lunghi mesi in cui sono stata, come milioni di Europei, privata di luci della ribalta o di schermi cinematografici, passando, quando era possibile uscire, di fonte ai teatri chiusi...

Le Por do Sol di. Jeri sono una meraviglia sempre rinnovata, e che, proprio perché condivisa con un gan numero di persone, in una socialità spontanea, ci fa sentire pienamente umani, accomunati dal senso della bellezza e della vita.

\footnotetext{
${ }^{8}$ Titolo di un libro di Karl Nemeth, Lockdownj, Der Corona Wahnsinn, pubblicato in Germania. Espressione molto utilizzata nei paesi di lingua tedesca, dove l'opposizione ai lockdon è molto forte.

${ }^{9}$ O Dia Nacional de Zumbi e da Consciência Negra, celebrato il 20 di novembre, fu instituito ufficialmente con la Lei no 12.519, del 10 di novembre del 2011. La data ricorda la morte di Zumbi, leader del Quilombo dos Palmares - nella Regione Nordeste del Brasile. I Quilombo erano aree e villaggi dove si rifugiavano gli schiavi fuggiaschi. Zumbi fu ucciso nel 1695. resistência à escravidão no Brasil.

${ }^{10} \mathrm{https}$ //brasilescola.uol.com.br/datas-comemorativas/dia-nacional-da-consciencia-negra.htm
} 
Figura 3. Tramonto a Jeri.

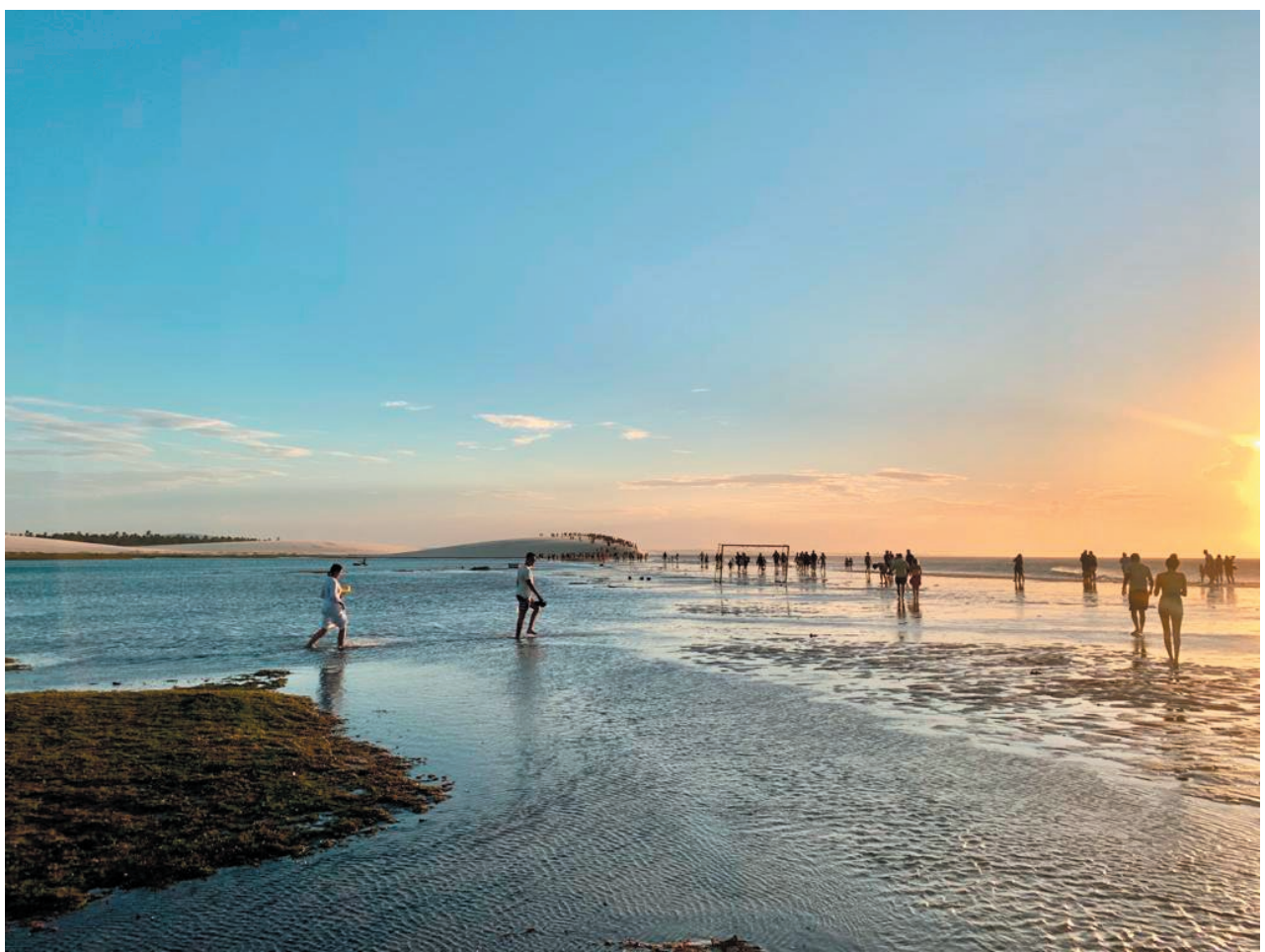

DATI Coronavirus per lo stato del CEARA al 4 febbraio 2021:

Il Ceara ha 9.187.103 abitanti (stimati); 378.000 casi, 288.000 guarigioni, 10.556 morti. Il Belgio ha 11.460.000 abitanti; 716.000 casi -49.302 guarigioni- 21.216 morti. ${ }^{11}$ Il Belgio ha praticato rigidissimi lockdown.

Il Portogallo ha 10.280.000, casi 741.000, guariti 563.000, 13.257 morti

Il Portogallo ha praticato rigidi lockdown rigido.

Dati ricavati da:

https://covid.saude.gov.br

https://www.worldometers.info/coronavirus/country/brazil/

\section{A felicidade è uma barraca de praia. Le spiagge democratiche.}

Le spiagge di Rio de Janeiro sono tra le più famose e le più belle del mondo, nella loro unica combinazione con colline arrotondate. I loro nomi sono diventati il simbolo di

${ }^{11}$ https://www.worldometers.info/coronavirus/country/belgium/ 
Figura 4. Gente in spiaggia

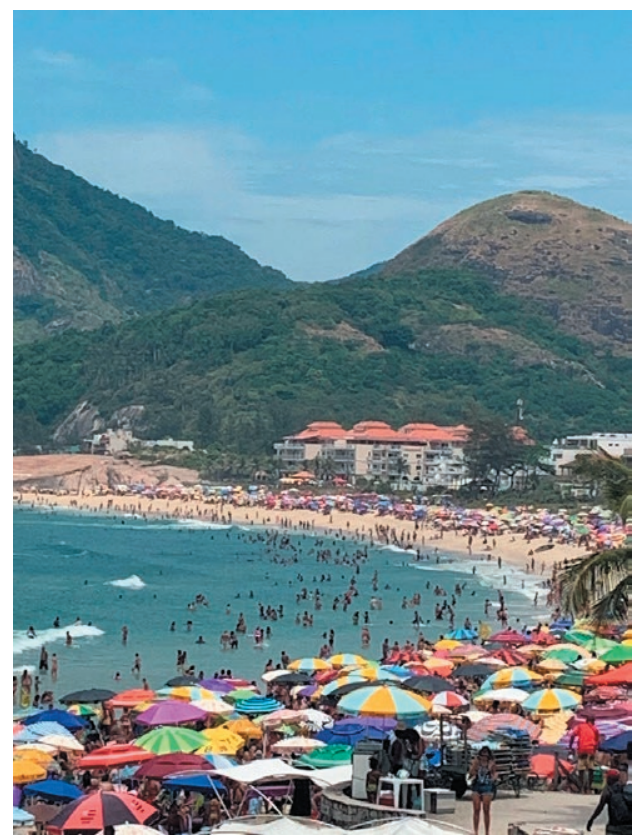

Figura 5. Negozio in spiaggia.

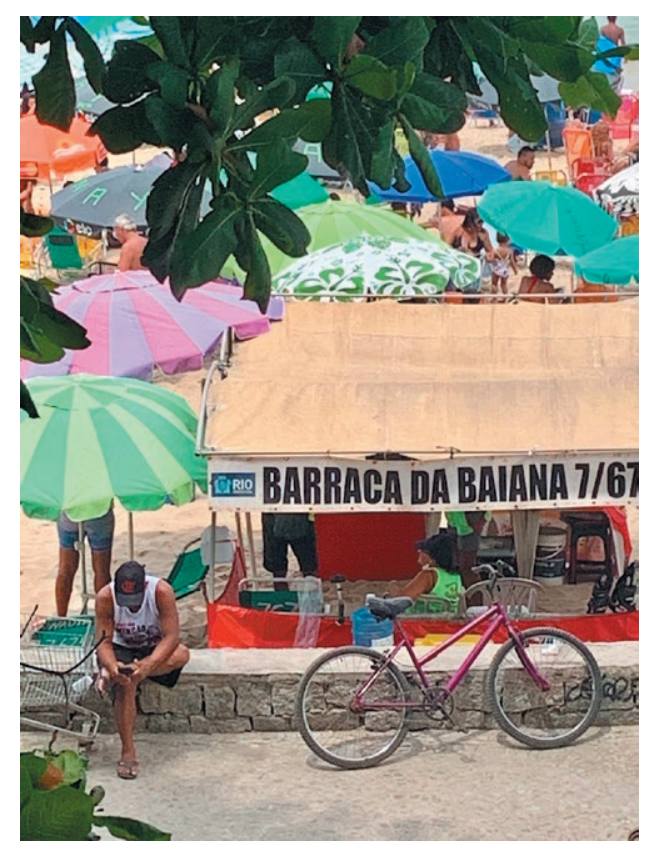

una vita meravigliosa, come la città di Rio, "Cidade maravilhosa": Copacabana, Ipanema, Leblon, Sao Conrado.

A Rio, come in tutto il Brasile, "as praias são um bens naturais e públicos", mi ha spiegato la receptionist del primo hotel dove ho alloggiato. Le spiagge sono pubbliche, appartengono a tutti...sono dunque democratiche. In un paese di grandi diseguaglianze, nessuno ha osato privatizzare le spiagge. Sono convinta che non riuscirà ad appropriarsene nemmeno la nuova aristocrazia neo-feudale dell' $1 \%$ - che si compra isole e vola in jet privato - mentre i comuni mortali non possono viaggiare e le compagnie aeree fanno fallimento. Le spiagge di Rio resteranno di tutti. I ragazzini della favela di Vidigal continueranno a scendere verso a Leblon a a Ipanema, bagnandosii accanto all'Hotel Fasano dove sosta Lady Gaga.

Le spiagge sono il centro della vita sociale per le famiglie, i gruppi amici, gli studenti, le coppie, giovani innamorati e anziani malfermi.

Le spiagge sono anche la fonte di reddito per migliaia e migliaia di persona, prima di tutto per i gestori delle "Barracas", poi per i venditori ambulanti.

Che cosa sono le "barracas"? Per il dizionario barraca é: "Pequeno abrigo de madeira, lona, plástico etc.” oppure "Fam. Grande guarda-sol de praia." Le "barracas” delle spiagge sono piccole strutture di legno e plastica, nelle quali lavorano tre o quattro persone, che affittano sedie e ombrelloni (5-7 reais per una sedia, 10 per un ombrellone), proponendo bevande fresche, dal cocco gelato alla birra. Le più organizzate preparano anche le caipirinhe, le caipivodka e altri cocktails.

Ogni barraca ha un proprietario ed un nome, che è quasi sempre quello dello stesso proprietario, raramente di un club di football o di una località. Nomi? piuttosto, soprannomi: 
almeno per la barraca di Miao, quella di Dudu padrino \& Gil (Il Padrino è proprio quello di Coppola, visto che cee un poster di Marlon Brando attaccato al telone), di Eddy Murphy, del Russo, di Gravidinho (personaggio infantile dei fumetti). Più tranquilli i nomi delle signore: Tia Ana, Baiana, Sonia, Reinha (la cui barraca è o Paraiso do Leblon). Non mancano le coppie: Maria e Caio. O ancora Paula e Cicero (eh si...Cicero, come padre Cicero).

\section{All the world's a stage (William Shakespeare)}

Le spiagge di Rio non sarebbero le spiagge di Rio senza i venditori ambulanti che passano con la frequenza di due per minuto. Attori spontanei, i venditori variano le modulazioni della voce e la gestualità, mentre i professionisti completano lo spettacolo con l'abbigliamento di funzione. In quest'ultima categoria vanno annoverati i venditori di "salgado arabe" Sheik, della "deliciosa comoda arabe" o del kebab in djellabas bianche o verdi, e quelli del Matte Leao gelato, in giacca gialla con il Leone sulla schiena (vedi infra). "Camarao, camarao" grida uno ragazzo che tiene un vassoio di spiedini di gamberi con l'eleganza di un cameriere di Chez MAXIM, seguito a ruota dal servizio bevande, Hello cerveja...cerveja...cerveja...diviso in diverse specializzazioni, "Corona gelada...corona gelada" ma anche Heineken Heineken. Per chi non beve alcolici si moltiplica l'offerta di diversi tipi di Matte o limonate, in contenitori di latta, che paiono pesanti sulle spalle dei venditori, spesso anziani. Uno di questi, ormai privo di voce, utilizza una registrazione che ripete con tono monotono: Temos mate e limonada. Aceptamos cartones. Il Mate può essere industriale o artigianale.

Il Mate Leao è una marca storica12: "A empresa foi fundada por Agostinho Ermelino de Leão Junior, em 8 de maio de 1901, como uma companhia de industrialização de ervamate no Paraná. Por isso, foi batizada Leão Junior."

Tradizionalmente il Mate si consumava caldo. Negli anni 1950, a Rio de Janeiro, si prese l'abitudine di consumarlo ghiacciato e di berlo in spiaggia, fabbricato artigianalmente. Negli anni 80 , la marca Leao ne fece una bibita ${ }^{13}$.

Assieme al mate, si vendono i biscotti Globo, i più famosi di Rio. Per chi ha un appetito un po' più importante ci sono i venditori di "queso cohalio", un formaggio grigliato direttamente in spiaggia su appositi fornelli. Poi ci sono i carrettoni che vendono il Milho...Milho cozidho, mais cotto, sgranato e servito in un piatto. Per il dessert, ci sono i gelati KIBON, ed il famoso sorvete Italia.

I generi non alimentari comprendono i pareo, le camice a fiori, gli occhiali, e, soprattutto i bikini, che appesi ad una specie di albero della cuccagna, si muovono sopra gli ombrelloni, come dotatisi vita propria. Non vé dubbio che l' albero dei bikini sia una delle più singolari "performances" dei venditori delle spiagge di Rio, girandola di colori impressi su minuscoli pezzi di tessuto.

Il bikini carioca è minimalista, lascia visibili i corpi, tutti i corpi, quelli delle le "mulheres esculturais", ma anche quelli di donne e uomini "com estrias, celulites e barriguinhas indesejáveis”,(Goldenberg, 2002, p.8).

\footnotetext{
${ }^{12}$ https://www.cocacolabrasil.com.br/historias/12-curiosidades-sobre-o-matte-leao

${ }^{13} \mathrm{Nel} 2007$ la ditta Leao fu acquistata dalla Coca Cola. "Em 2007, passou a integrar o Sistema Coca-Cola Brasil e, em 2012, após a conclusão do processo de incorporação, ganhou o nome oficial de Leão Alimentos e Bebidas."
} 
Figura 6. Bagnanti, mare e spiaggia.

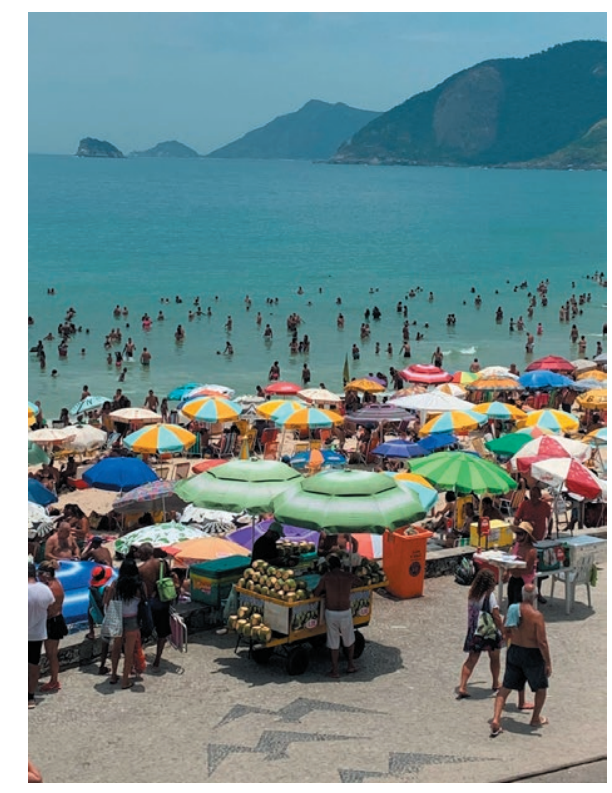

\section{0 corpo como cultura}

Lo spettacolo dei corpi sulle spiagge di Rio non può lasciare indifferenti: "Branco, moreno, mulato ou negro, nu e vestido, o corpo carioca provoca uma verdadeira explosão de significados, como queria Malinowski, revelando as especificidades da cultura da 'Cidade Maravilhosa"' (Goldberg, 2002, p. 7).

Tra i significati multipli del corpo carioca, vi è l'articolazione tra la specificità della città che ne fa "um espaço privilegiado para estudar o atual culto ao corpo" (p. 8), ed una tendenza più generale inscritta nella cultura narcisista della modernità. Ma la convivenza "aparentemente tranqüila" entre "corpos bronzeados, musculosos, magros, altos", di "mulheres esculturais", di donne e uomini "com estrias, celulites e barriguinhas indesejáveis",(Goldenberg, 2002, p.8) costituisce un "corpo carioca" che è "corpo seminu, praia, sol, carnaval, festa, juventude, liberdade, sexualidade, alegria, irreverência, descontração, humor, informalidade, criatividade, hedonismo" (Goldberg, 2002, p. 7).

In queste poche pagine non è possibile trattare, anche in maniera superficiale la questione del corpo, che ci condurrebbe lontano, verso Exu, o senior do corpo, o masculino, e Pomba-gira, o feminino nei canti dell'umbanda.

\section{Conclusioni: Brasil Land von Zukunft?}

Luis Antonio Simas scrive: "No gosto di Brasil (inteso come stato prodotto dalla colonizzazione). Eu gosto da brasilidade, essa comunidade de sentidos, afetos, sonoridades, rasuras, contradiçoes, naufragios, ilhas fugidias, identidades, inviaveis, subversões coti- 
dianas, voo de arara e picada de maribondo, saravá e samba." (Simas, 2020, p. 177)...una brasilianità fatta di una adeguazione trasgressiva di fronte alla colonizzazione, al potere... ai suoi assurdi ordini e alla sua profonda violenza. Anche nel tragico periodo che stiamo vivendo, la brasilidade dimostra questo: l'adeguazione trasgressiva...

DATI Coronavirus per lo stato di Rio de Janeiro al 4 febbraio 2021:

Lo stato di Rio de Janeiro ha 17.366.189 Casi 532.000 guariti 497.000 morti 30.354

Lo stato di Rio de Janeiro non ha mai confinato.

L'Italia ha 60.000.000 di abitanti, ha avuto 2,6000.000 casi, guariti 2.008.000, morti 90.241.000

L'Italia ha confinato in maniera durissima la popolazione in primavera e dall'autunno fino ad oggi.

Fonti:

Dati ricavati da:

https://covid.saude.gov.br

https://www.worldometers.info/coronavirus/country/brazil/

\section{Bibliografia}

Goldenberg, M. et al. (Org.) (2002). Nu \& Vestido: dez antropólogos revelam a cultura do corpo carioca. Rio de Janeiro: Record.

Nemeth, K. (2020). LOCKDOWN: Der Corona Wahnsinn. Germania: Independently Published.

Simas, L.A., Rufino, L., Haddock-Lobo, R. (2020). Arruaças, uma filosofia popular brasileira. Rio de Janeiro: Bazar do Tempo. 